\title{
EXISTENCE AND UNIQUENESS OF SOLUTIONS TO DISTRIBUTIONAL DIFFERENTIAL EQUATIONS INVOLVING HENSTOCK-KURZWEIL-STIELTJES INTEGRALS
}

\author{
GUOJU YE, MINGXIA ZHANG, WEI LIU, AND DAFANG ZHAO
}

\begin{abstract}
This paper is concerned with the existence and uniqueness of solutions to the second order distributional differential equation with Neumann boundary value problem via Henstock-Kurzweil-Stieltjes integrals. The existence of solutions is derived from Schauder's fixed point theorem, and the uniqueness of solutions is established by Banach's contraction principle. Finally, two examples are given to demonstrate the main results.
\end{abstract}

\section{INTRODUCTION}

In this paper we apply the Henstock-Kurzweil-Stieltjes integral to establish the existence and uniqueness of solutions to the second order distributional differential equation (in short DDE)

$$
-D^{2} x=f(t, x)+g(t, x) D u, \quad t \in[0,1],
$$

subject to the Neumann boundary value condition

$$
D x(0)=D x(1)=0,
$$

where $D, D^{2}$ stand for the first order and the second order distributional derivative, respectively, $x$ and $u$ are regulated functions (see Definition 2.2) such that both are left-continuous on $(a, b]$ and right-continuous at $a$, the function $f(\cdot, x(\cdot))$ is Henstock-Kurzweil integrable, and $g(\cdot, x(\cdot))$ is a function of bounded variation.

We know that regulated functions contain continuous functions and functions of bounded variation as special cases. Especially, when $u$ is an absolutely continuous function, its distributional derivative is the usual derivative and we obtain the ordinary differential equation (in short ODE); when $u$ is a function of bounded variation, $D u$ can be identified with a Stieltjes measure, and 1.1 is called measure differential equation (in short MDE). Results concerning existence, uniqueness and

2010 Mathematics Subject Classification. 34A37; 34B15; 26A42.

Key words and phrases. Second-order distributional differential equation; Neumann boundary value problem; Henstock-Kurzweil-Stieltjes integral.

This work was partially supported by the National Key Research and Development Program of China (2018YFC1508106), the Natural Science Foundation of Jiangsu Province (No. BK20180500); and the Fundamental Research Funds for the Central Universities (Grant Nos. 2017B19714, 2017B07414). 
stability of solutions for MDEs have been extensively studied in many papers, see [2, 11]. For example, in [2], the authors establish the existence of solutions for the first order MDE

$$
D x=f(t, x)+G(t, x) D u .
$$

Recently, in [8], authors obtained the existence result for the first order DDE of the form

$$
D x=f(t, x) D u .
$$

As far as we know, few papers are concerned with the second order DDE. The purpose of this paper is to fill this gap, considering the second order DDE with the Neumann boundary value problem.

Neumann boundary value problems have played an important role in mathematical physics, and hence have attracted the attention of many researchers. Under suitable conditions, some existence and uniqueness results and the multiplicity of positive solutions for the Neumann boundary value problem have been established. For example, in [1], a Lyapunov-type inequality and Schauder's fixed point theorem were used to obtain existence and uniqueness results for the Neumann boundary value problem as

$$
-u^{\prime \prime}(x)=f(x, u(x)), \quad x \in[0, L], \quad u^{\prime}(0)=u^{\prime}(L)=0 .
$$

In [13, by using a fixed point theorem in a cone, the authors established the existence and multiplicity of the positive solutions to the second order Neumann boundary value problem $\sqrt{1.3}$ with parameter. Particularly, problem $\sqrt{1.3}$ is a special case of problem (1.1)-(1.2).

In order to deal with the problem (1.1)- 1.2 , we apply the Henstock-KurzweilStieltjes integral, which is a powerful tool for the study of DDEs and contains the Henstock-Kurzweil integral ([6, 12, 9, [10]), the regulated primitive integral (14]), the Lebesgue-Stieltjes integral, and the Riemann-Stieltjes integral. With the help of Henstock-Kurzweil-Stieltjes integrals, we establish the existence and uniqueness of solutions for the second order DDE with Neumann boundary value problem. Therefore, our results can be seen as a generalization of the results in [1, 13].

This paper is organized as follows. In Section 2, we recall the definition and some basic properties of Henstock-Kurzweil-Stieltjes integrals and regulated functions. In Section 3, two results of the existence and uniqueness of solutions are established by using Schauder's fixed point theorem and Banach's contraction principle. In Section 4, we give two examples to illustrate Theorem 3.4 and Theorem 3.6 .

\section{Preliminaries}

2.1. Regulated functions. In this subsection, we recall the definition and some basic properties of regulated functions. First, we introduce functions of bounded variation, which are a special case of regulated functions.

We consider a nondegenerate compact interval $[a, b] \subset \mathbb{R}$, and denote by $\mathcal{D}_{a, b}$ the set of all divisions of the form

$$
d=\left\{t_{0}, \ldots, t_{m}\right\}, \quad a=t_{0}<t_{1}<\cdots<t_{m}=b .
$$


Definition 2.1 ([5]). For a given function $g:[a, b] \rightarrow \mathbb{R}$ and a given division $d \in \mathcal{D}_{a, b}$ of the form [2.1, we define the total variation $\underset{[a, b]}{\operatorname{Var}} g$ of $g$ by

$$
\operatorname{Var}_{[a, b]} g:=\sup _{d \in \mathcal{D}_{a, b}} \sum_{j=1}^{m}\left|g\left(t_{j}\right)-g\left(t_{j-1}\right)\right| .
$$

The set of functions of bounded variation on $[a, b]$ is denoted by

$$
B V([a, b] ; \mathbb{R}):=\left\{g:[a, b] \rightarrow \mathbb{R} ; \operatorname{Var}_{[a, b]} g<\infty\right\} .
$$

Definition $2.2([3])$. A function $f:[a, b] \rightarrow \mathbb{R}$ is called regulated on $[a, b]$ if the one sided-limits exist, more precisely:

$$
\lim _{s \rightarrow t-} f(s)=: f(t-), \quad t \in(a, b] \quad \text { and } \quad \lim _{s \rightarrow t+} f(s)=: f(t+), \quad t \in[a, b),
$$

exist, with the convention

$$
f(a-)=f(a), \quad f(b+)=f(b) .
$$

We denote by $G([a, b] ; \mathbb{R})$ the set of all regulated functions $f:[a, b] \rightarrow \mathbb{R}$ and by $G_{-}([a, b] ; \mathbb{R})$ the set of all left-continuous regulated functions on $(a, b]$ and rightcontinuous at $a$. The space $G([a, b] ; \mathbb{R})$ endowed with the norm

$$
\|f\|=\sup _{t \in[a, b]}|f(t)|
$$

is a Banach space, and $G_{-}([a, b] ; \mathbb{R})$ is a closed subspace of $G([a, b] ; \mathbb{R})$.

We know that the function $f$ is regulated if and only if it is a uniform limit of a sequence of step functions, and we denote by $S([a, b] ; \mathbb{R})$ the set of all step functions $w:[a, b] \rightarrow \mathbb{R}$.

We now list some basic properties of the above spaces which we will need later.

Lemma 2.3 ([5]).

(i) The sets $S([a, b] ; \mathbb{R}), B V([a, b] ; \mathbb{R})$, and $G([a, b] ; \mathbb{R})$ are vector spaces satisfying the inclusion

$$
S([a, b] ; \mathbb{R}) \subset B V([a, b] ; \mathbb{R}) \subset G([a, b] ; \mathbb{R}) .
$$

(ii) For every $f \in G([a, b] ; \mathbb{R})$ and $\varepsilon>0$ there exists $w \in S([a, b] ; \mathbb{R})$ such that $\|f-w\| \leq \varepsilon, w(t) \in \bigcup_{\tau \in[a, b]}\{f(\tau)\}$ for every $t \in[a, b], \operatorname{Var}_{[a, b]} w \leq \operatorname{Var}_{[a, b]} f$.

The next result is important when investigating compactness in $G([a, b] ; \mathbb{R})$.

Definition 2.4 ([3]). A set $\mathbb{M} \subset G([a, b] ; \mathbb{R})$ is said to be equiregulated if, for every $\varepsilon>0$ and $t_{0} \in[a, b]$, there exists $\delta>0$ such that for every $x \in \mathbb{M}$ and $t \in[a, b]$ we have

(i) if $t_{0}-\delta<t<t_{0}$, then $\left|x\left(t_{0}-\right)-x(t)\right|<\varepsilon$;

(ii) if $t_{0}<t<t_{0}+\delta$, then $\left|x(t)-x\left(t_{0}+\right)\right|<\varepsilon$.

Lemma 2.5 ([3, Corollary 2.4]). A set $\mathbb{M} \subset G([a, b] ; \mathbb{R})$ is relatively compact if and only if it is equiregulated and for every $t \in[a, b]$ the set $\{x(t): x \in \mathbb{M}\}$ is bounded in $\mathbb{R}$. 
2.2. The Henstock-Kurzweil-Stieltjes integral. In this subsection, we recall some basic properties of the Henstock-Kurzweil-Stieltjes integral. We cite most of the results without proof, and interested readers can find more information in [6, 12, 7, 9, 10].

We consider a division $d=\left\{t_{0}, \ldots, t_{m}\right\} \in \mathcal{D}_{a, b}$ from $(2.1)$, and define a partition $D$ as

$$
D=\left\{\left(\tau_{j},\left[t_{j-1}, t_{j}\right]\right) ; j=1, \ldots, m\right\} ; \quad \tau_{j} \in\left[t_{j-1}, t_{j}\right], \quad \forall j=1, \ldots, m .
$$

The basic concept in the Henstock-Kurzweil-Stieltjes integration theory is a $\delta$-fine partition.

We define the set

$$
\Gamma(a, b):=\{\delta:[a, b] \rightarrow \mathbb{R} \mid \delta(t)>0 \text { for every } t \in[a, b]\} .
$$

An element $\delta \in \Gamma(a, b)$ is called a gauge.

Definition 2.6 ([7]). Let $\delta \in \Gamma(a, b)$ be a given gauge. A partition $D$ of the form 2.2 is said to be $\delta$-fine if for every $j=1, \ldots, m$ we have

$$
\tau_{j} \in\left[t_{j-1}, t_{j}\right] \subset\left(\tau_{j}-\delta\left(\tau_{j}\right), \tau_{j}+\delta\left(\tau_{j}\right)\right),
$$

and $\tau_{1}=a, \tau_{m}=b$. The set of all $\delta$-fine partitions is denoted by $\mathcal{F}_{\delta}(a, b)$.

For given functions $f, g:[a, b] \rightarrow \mathbb{R}$ and a partition $D$ of the form (2.2), we define the Henstock-Kurzweil-Stieltjes integral sum $K_{D}(f, g)$ by the formula

$$
\begin{aligned}
K_{D}(f, g) & =\sum_{j=1}^{m} f\left(\xi_{j}\right)\left(g\left(t_{j}\right)-g\left(t_{j-1}\right)\right) \\
& =f(b) g(b)-f(a) g(a)-\sum_{j=1}^{m}\left(f\left(\xi_{j}\right)-f\left(\xi_{j-1}\right)\right) g\left(t_{j-1}\right),
\end{aligned}
$$

in which $\xi_{0}=a, \xi_{m}=b$.

Definition $2.7([7])$. Let $f, g:[a, b] \rightarrow \mathbb{R}$ be given. We say that $J \in \mathbb{R}$ is the Henstock-Kurzweil-Stieltjes integral over $[a, b]$ of $f$ with respect to $g$ and is denoted by

$$
J=\int_{a}^{b} f(t) d g(t)=\int_{a}^{b} f d g,
$$

if for every $\varepsilon>0$ there exists $\delta \in \Gamma(a, b)$ such that for every $D \in \mathcal{F}_{\delta}(a, b)$, we have

$$
\left|J-K_{D}(f, g)\right| \leq \varepsilon .
$$

Particularly, if $g(t) \equiv t$, then Definition 2.7 is the definition of HenstockKurzweil integrals (HK-integrals for short).

Remark 2.8. According to [7, we know that the Henstock-Kurzweil-Stieltjes integral is a generalization of the Lebesgue-Stieltjes integral and the Riemann-Stieltjes integral. For example, when $g$ is a regulated function, the function $f$ is not Lebesgue-Stieltjes integrable with respect to the function $g$. Also, the RiemannStieltjes integral will not be appropriate when the function $f$ and the function $g$ have a common point of discontinuity. 
Lemma 2.9 ([5, Corollary 3.10.]).

(i) If $f \in G([a, b] ; \mathbb{R})$ and $g \in B V([a, b] ; \mathbb{R})$ then the integral $\int_{a}^{b} f(t) d g(t)$ exists and

$$
\left|\int_{a}^{b} f d g\right| \leq\|f\| \operatorname{Var}_{[a, b]} g .
$$

(ii) If $f \in B V([a, b] ; \mathbb{R})$ and $g \in G([a, b] ; \mathbb{R})$ then the integral $\int_{a}^{b} f(t) d g(t)$ exists and

$$
\left|\int_{a}^{b} f d g\right| \leq\left(|f(a)|+|f(b)|+\operatorname{Var}_{[a, b]} f\right)\|g\| .
$$

The proof of the following proposition is similar to that for the Young integral in [5. Proposition 3.12]; however, in order to make the paper self-contained we give the proof here.

Proposition 2.10. Consider $f, f_{n} \in G([a, b] ; \mathbb{R}), g, g_{n} \in B V([a, b] ; \mathbb{R})$ for $n \in \mathbb{N}$ such that

$$
\lim _{n \rightarrow \infty}\left\|f-f_{n}\right\|=0, \quad \lim _{n \rightarrow \infty}\left\|g-g_{n}\right\|=0, \quad \sup _{n \in \mathbb{N}[a, b]} \operatorname{Var}_{n} \leq C<\infty .
$$

Then

$$
\lim _{n \rightarrow \infty} \int_{a}^{b} g_{n}(t) d f_{n}(t)=\int_{a}^{b} g(t) d f(t) .
$$

Proof. For any $\varepsilon>0$, there exists $w \in S([a, b] ; \mathbb{R}) \subseteq B V([a, b] ; \mathbb{R})$, so $\underset{[a, b]}{\operatorname{Var}} w=C_{1}<$ $\infty$, such that $\|f-w\|<\varepsilon$, and there exists $N>0$ for which $\left\|f-f_{n}\right\|<\varepsilon$ and $\left\|g-g_{n}\right\|<\varepsilon$ when $n>N$. Then we obtain by Lemma 2.3 and Lemma 2.9 that

$$
\begin{array}{rl}
\mid \int_{a}^{b} & g(t) d f(t)-\int_{a}^{b} g_{n}(t) d f_{n}(t) \mid \\
= & \mid \int_{a}^{b} g(t) d f(t)-\int_{a}^{b} g(t) d w(t)+\int_{a}^{b} g(t) d w(t)-\int_{a}^{b} g_{n}(t) d w(t) \\
& +\int_{a}^{b} g_{n}(t) d w(t)-\int_{a}^{b} g_{n}(t) d f(t)+\int_{a}^{b} g_{n}(t) d f(t)-\int_{a}^{b} g_{n}(t) d f_{n}(t) \mid \\
\leq & \left|\int_{a}^{b}\left(g(t)-g_{n}(t)\right) d(f(t)-w(t))\right|+\left|\int_{a}^{b}\left(g(t)-g_{n}(t)\right) d w(t)\right| \\
& +\left|\int_{a}^{b} g_{n}(t) d\left(f(t)-f_{n}(t)\right)\right| \\
\leq & \left(\left|g(a)-g_{n}(a)\right|+\left|g(b)-g_{n}(b)\right|+\operatorname{Var}_{[a, b]}\left(g-g_{n}\right)\right)\|f-w\|+\left\|g-g_{n}\right\| \underset{[a, b]}{\operatorname{Var} w} \\
& +\left(\left|g_{n}(a)\right|+\left|g_{n}(b)\right|+\operatorname{Var}_{[a, b]} g_{n}\right)\left\|f-f_{n}\right\|
\end{array}
$$




$$
\begin{aligned}
& \leq 2\left(\left\|g-g_{n}\right\|+C\right)\|f-w\|+\left\|g-g_{n}\right\| \underset{[a, b]}{\operatorname{Var}} w+\left(2\left\|g_{n}\right\|+C\right)\left\|f-f_{n}\right\| \\
& \leq\left[2(\varepsilon+C)+C_{1}+\left(2\left\|g_{n}\right\|+C\right)\right] \varepsilon .
\end{aligned}
$$

Hence,

$$
\lim _{n \rightarrow \infty} \int_{a}^{b} g_{n}(t) d f_{n}(t)=\int_{a}^{b} g(t) d f(t)
$$

This completes the proof.

For the HK-integral, we have the following controlled convergence theorem, which will be used later.

Lemma 2.11 ([6, Theorem 7.1]). If the following conditions are established:

(i) $f_{n}(n=1,2, \ldots)$ is HK-integrable on $[a, b]$ and $f_{n}(t) \rightarrow f(t)$ a.e., for all $t \in[a, b]$

(ii) $g, h$ are $H K$-integrable on $[a, b]$ and $g(t) \leq f_{n}(t) \leq h(t)$ for every $n$ a.e., for all $t \in[a, b]$;

then $f$ is HK-integrable on $[a, b]$ and

$$
\lim _{n \rightarrow \infty} \int_{a}^{b} f_{n}(t) d t=\int_{a}^{b} f(t) d t
$$

\section{MAin RESUlts}

In this section, let

$$
B_{r}=\left\{x \in G_{-}([0,1] ; \mathbb{R}):\|x\| \leq r\right\}, \quad r>0 .
$$

We assume that the functions $f, g$ in problem $1.1-1.2$ satisfy the following assumptions:

$\left(\mathrm{C}_{1}\right) f(\cdot, x(\cdot))$ is HK-integrable for all $x \in B_{r}$;

$\left(\mathrm{C}_{2}\right) f(t, \cdot)$ is continuous for all $t \in[0,1]$;

$\left(\mathrm{C}_{3}\right)$ there exists $q_{1}, q_{2} \in H K$ such that $q_{1}(\cdot) \leq f(\cdot, x(\cdot))+x(\cdot) \leq q_{2}(\cdot)$ for all $x \in B_{r}$

$\left(\mathrm{C}_{4}\right) g(\cdot, x(\cdot))$ is a function of bounded variation with $g(0, x(0))=g_{0} \in \mathbb{R}$ for all $x \in B_{r}$

$\left(\mathrm{C}_{5}\right) g(t, \cdot)$ is continuous on $[0,1]$, uniformly for all $t \in[0,1]$, i.e., for every $\varepsilon>0$, there exists $\delta>0$ such that if $\left|s_{1}-s_{0}\right|<\delta$ then $\left|g\left(t, s_{1}\right)-g\left(t, s_{0}\right)\right|<\varepsilon$ for all $t \in[0,1]$;

$\left(\mathrm{C}_{6}\right)$ there exists a constant $\bar{g}>0$ such that $\underset{[0,1]}{\operatorname{Var}} g(\cdot, x(\cdot)) \leq \bar{g}$ for all $x \in B_{r}$.

Lemma 3.1. Let the functions $f, g$ satisfy the assumptions $\left(C_{1}\right)-\left(C_{6}\right)$. A function $x:[0,1] \rightarrow \mathbb{R}$ is a solution of problem (1.1)-(1.2) on $[0,1]$ if and only if $x$ satisfies the integral equation

$$
x(t)=\int_{0}^{1} K(t, s)(f(s, x(s))+x(s)) d s+\int_{0}^{1} K(t, s) g(s, x(s)) d u(s),
$$


where

$$
K(t, s)= \begin{cases}\frac{\left(e^{2-(t+s)}+e^{2-(t-s)}+e^{t+s}+e^{t-s}\right)}{2\left(e^{2}-1\right)}, & 0 \leq s \leq t \leq 1 \\ \frac{\left(e^{2-(s+t)}+e^{2-(s-t)}+e^{s+t}+e^{s-t}\right)}{2\left(e^{2}-1\right)}, & 0 \leq t \leq s \leq 1 .\end{cases}
$$

Proof. Assume first that $x:[0,1] \rightarrow \mathbb{R}$ is a solution of problem 1.1 - 1.2 . Then

$$
-D^{2} x+D x-D x+x=f(t, x(t))+g(t, x(t)) D u+x, \quad t \in[0,1] .
$$

Multiplying both sides of $(3.3)$ by $e^{-t}$ and integrating, we obtain

$$
-e^{-t}(D x+x)=\int_{0}^{t} e^{-s}(f(s, x(s))+x(s)) d s+\int_{0}^{t} e^{-s} g(s, x(s)) d u(s)+c
$$

for some constant $c$, and so

$$
D x=\int_{0}^{t}-e^{t-s}(f(s, x(s))+x(s)) d s+\int_{0}^{t}-e^{t-s} g(s, x(s)) d u(s)-c e^{t}-x .
$$

By the boundary condition $D x(0)=0$, we have that $c=-x(0)$. Now, multiplying both sides of (3.4) by $e^{2 t}$, we get

$$
-e^{t}(D x+x)+e^{2 t} x(0)=\int_{0}^{t} e^{2 t-s}(f(s, x(s))+x(s)) d s+\int_{0}^{t} e^{2 t-s} g(s, x(s)) d u(s) .
$$

Integrating (3.6) from 0 to $\tau$, we obtain

$$
\begin{aligned}
& -e^{\tau} x(\tau)+\frac{1}{2}\left(e^{2 \tau}+1\right) x(0) \\
& =\int_{0}^{\tau} \int_{0}^{t} e^{2 t-s}(f(s, x(s))+x(s)) d s d t+\int_{0}^{\tau} \int_{0}^{t} e^{2 t-s} g(s, x(s)) d u(s) d t .
\end{aligned}
$$

Therefore, by the Tonelli and the Fubini theorems, we have that

$$
\begin{aligned}
& -e^{\tau} x(\tau)+\frac{1}{2}\left(e^{2 \tau}+1\right) x(0) \\
& =\int_{0}^{\tau} \frac{1}{2}\left(e^{2 \tau-s}-e^{s}\right)(f(s, x(s))+x(s)) d s+\int_{0}^{\tau} \frac{1}{2}\left(e^{2 \tau-s}-e^{s}\right) g(s, x(s)) d u(s) .
\end{aligned}
$$

Moreover, substituting $t=1$ into 3.5 and $\tau=1$ into 3.8 , one has

$$
-e x(1)+e^{2} x(0)=\int_{0}^{1} e^{2-s}(f(s, x(s))+x(s)) d s+\int_{0}^{1} e^{2-s} g(s, x(s)) d u(s)
$$

and

$$
\begin{aligned}
& -e x(1)+\frac{1}{2}\left(e^{2}+1\right) x(0) \\
& =\int_{0}^{1} \frac{1}{2}\left(e^{2-s}-e^{s}\right)(f(s, x(s))+x(s)) d s+\int_{0}^{1} \frac{1}{2}\left(e^{2-s}-e^{s}\right) g(s, x(s)) d u(s) .
\end{aligned}
$$


According to 3.9 and 3.10 , one has

$$
x(0)=\int_{0}^{1} \frac{e^{2-s}+e^{s}}{e^{2}-1}(f(s, x(s))+x(s)) d s+\int_{0}^{1} \frac{e^{2-s}+e^{s}}{e^{2}-1} g(s, x(s)) d u(s) .
$$

Substitute (3.11) into 3.8), we have

$$
\begin{aligned}
x(t)= & \int_{0}^{1} \frac{\left(e^{2 t}+1\right)\left(e^{2-s}+e^{s}\right)}{2\left(e^{2}-1\right) e^{t}}(f(s, x(s))+x(s)) d s \\
& +\int_{0}^{t} \frac{e^{s}-e^{2 t-s}}{2 e^{t}}(f(s, x(s))+x(s)) d s \\
& +\int_{0}^{1} \frac{\left(e^{2 t}+1\right)\left(e^{2-s}+e^{s}\right)}{2\left(e^{2}-1\right) e^{t}} g(s, x(s)) d u(s) \\
& +\int_{0}^{t} \frac{e^{s}-e^{2 t-s}}{2 e^{t}} g(s, x(s)) d u(s) .
\end{aligned}
$$

This shows that 3.1 holds.

Conversely, it is not difficult to calculate that (1.1)- 1.2 hold by taking the derivative of both sides of 3.12 . This completes the proof.

Remark 3.2. The function $K:[0,1] \times[0,1] \rightarrow \mathbb{R}$ given in 3.2 is positive continuous and of bounded variation. Moreover, we have

$$
\frac{2 e}{e^{2}-1}=m \leq K(t, s) \leq M=\frac{e^{2}+1}{e^{2}-1} .
$$

The following statement is the well-known Schauder's fixed point theorem.

Lemma 3.3. Let $\mathbb{M}$ be a nonempty closed convex subset of a normal vector space $\mathbb{R}$. Let $\mathcal{T}$ be a continuous map of $\mathbb{M}$ into a compact subset $\mathbb{K}$ of $\mathbb{M}$. Then $\mathcal{T}$ has a fixed point.

With the help of Lemma 3.1 and Lemma 3.3, we can now prove the existence of solutions for the problem 1.1$)-(1.2)$.

Theorem 3.4. Under the assumptions $\left(C_{1}\right)-\left(C_{6}\right)$, there exists at least one solution of the problem (1.1) -1.2 .

Proof. By $\left(\mathrm{C}_{3}\right)$, there exist $q_{1}, q_{2} \in H K$ such that their primitives are continuous and bounded on $[0,1]$.

Let

$$
Q:=\max _{t \in[0,1]}\left\{\left|\int_{0}^{t} q_{1}(s) d s\right|+\left|\int_{0}^{t} q_{2}(s) d s\right|\right\} .
$$

Then, for every $t \in[0,1]$, we have by 3.13 that

$$
-M Q \leq \int_{0}^{t} K(t, s)(f(s, x(s))+x(s)) d s \leq M Q .
$$

We define an operator $\mathcal{A}: G_{-}([0,1] ; \mathbb{R}) \rightarrow G_{-}([0,1] ; \mathbb{R})$ satisfying

$$
\mathcal{A} x(t)=\int_{0}^{1} K(t, s)(f(s, x(s))+x(s)) d s+\int_{0}^{1} K(t, s) g(s, x(s)) d u(s) .
$$


Now we prove this theorem in three steps.

Step 1. There exists $r>0$ such that $\mathcal{A}\left(B_{r}\right) \subseteq B_{r}$.

Let

$$
r:=M Q+(4 M-2 m)\left(\left|g_{0}\right|+\bar{g}\right)\|u\| .
$$

For all $x \in B_{r}$, by 3.14 and 3.15 , we have

$$
\begin{aligned}
|\mathcal{A} x(t)| & \leq\left|\int_{0}^{1} K(t, s)(f(s, x(s))+x(s)) d s\right|+\left|\int_{0}^{1} K(t, s) g(s, x(s)) d u(s)\right| \\
& \leq M Q+\left|\int_{0}^{1} K(t, s) g(s, x(s)) d u(s)\right| .
\end{aligned}
$$

By the Hölder inequality (Lemma 2.9p), one has

$$
\begin{aligned}
& \left|\int_{0}^{1} K(t, s) g(s, x(s)) d u(s)\right| \\
& \quad \leq(|K(t, 0) g(0, x(0))|+|K(t, 1) g(1, x(1))|+\underset{[0,1]}{\operatorname{Var} K(t, s) g(s, x(s)))\|u\|} \\
& \quad \leq\left(M\left|g_{0}\right|+M\left(\left|g_{0}\right|+\underset{[0,1]}{\operatorname{Var}} g(s, x(s))\right)+\left(\left|g_{0}\right|+\underset{[0,1]}{\operatorname{Var}} g(s, x(s))\right) \underset{[0,1]}{\operatorname{Var}} K(t, s)\right. \\
& \quad+M \underset{[0,1]}{\operatorname{Var}} g(s, x(s)))\|u\| \\
& \quad \leq(4 M-2 m)\left(\left|g_{0}\right|+\bar{g}\right)\|u\| .
\end{aligned}
$$

In view of 3.16) and (3.17), one has

$$
|\mathcal{A} x(t)| \leq M Q+(4 M-2 m)\left(\left|g_{0}\right|+\bar{g}\right)\|u\|=r .
$$

Therefore, $\mathcal{A}\left(B_{r}\right) \subseteq B_{r}$.

Step 2. $\mathcal{A}\left(B_{r}\right)$ is equiregulated.

For every $t_{0} \in[0,1), t \in\left[t_{0}, 1\right]$, and $x \in B_{r}$, we have

$$
\begin{aligned}
& \left|\mathcal{A} x(t)-\mathcal{A} x\left(t_{0}+\right)\right| \\
& \leq \mid \int_{0}^{1} \frac{\left(e^{2 t}+1\right)\left(e^{2-s}+e^{s}\right)}{2\left(e^{2}-1\right) e^{t}}(f(s, x(s))+x(s)) d s \\
& \quad-\int_{0}^{1} \frac{\left(e^{2 t_{0}+}+1\right)\left(e^{2-s}+e^{s}\right)}{2\left(e^{2}-1\right) e^{t_{0}+}}(f(s, x(s))+x(s)) d s \mid \\
& \quad+\mid \int_{0}^{1} \frac{\left(e^{2 t}+1\right)\left(e^{2-s}+e^{s}\right)}{2\left(e^{2}-1\right) e^{t}} g(s, x(s)) d u(s) \\
& \quad-\int_{0}^{1} \frac{\left(e^{2 t_{0}+}+1\right)\left(e^{2-s}+e^{s}\right)}{2\left(e^{2}-1\right) e^{t_{0}+}} g(s, x(s)) d u(s) \mid \\
& +\mid \int_{0}^{t} \frac{e^{s}-e^{2 t-s}}{2 e^{t}}(f(s, x(s))+x(s)) d s
\end{aligned}
$$




$$
\begin{aligned}
& -\int_{0}^{t_{0}+} \frac{e^{s}-e^{2\left(t_{0}+\right)-s}}{2 e^{t_{0}+}}(f(s, x(s))+x(s)) d s \mid \\
& +\left|\int_{0}^{t} \frac{e^{s}-e^{2 t-s}}{2 e^{t}} g(s, x(s)) d u(s)-\int_{0}^{t_{0}+} \frac{e^{s}-e^{2\left(t_{0}+\right)-s}}{2 e^{t_{0}+}} g(s, x(s)) d u(s)\right| \\
& \leq\left|\int_{0}^{1} \frac{\left(e^{t+\left(t_{0}+\right)}-1\right)\left(e^{t}-e^{t_{0}+}\right)\left(e^{2-s}+e^{s}\right)}{2\left(e^{2}-1\right) e^{t+\left(t_{0}+\right)}}(f(s, x(s))+x(s)) d s\right| \\
& +\left|\int_{0}^{1} \frac{\left(e^{t+\left(t_{0}+\right)}-1\right)\left(e^{t}-e^{t_{0}+}\right)\left(e^{2-s}+e^{s}\right)}{2\left(e^{2}-1\right) e^{t+\left(t_{0}+\right)}} g(s, x) d u(s)\right| \\
& +\mid \int_{0}^{t} \frac{e^{s}-e^{2 t-s}}{2 e^{t}}(f(s, x(s))+x(s)) d s \\
& -\int_{0}^{t_{0}+} \frac{e^{s}-e^{2 t-s}}{2 e^{t}}(f(s, x(s))+x(s)) d s \\
& +\int_{0}^{t_{0}+} \frac{e^{s}-e^{2 t-s}}{2 e^{t}}(f(s, x(s))+x(s)) d s \\
& -\int_{0}^{t_{0}+} \frac{e^{s}-e^{2\left(t_{0}+\right)-s}}{2 e^{t_{0}+}}(f(s, x(s))+x(s)) d s \mid \\
& +\mid \int_{0}^{t} \frac{e^{s}-e^{2 t-s}}{2 e^{t}} g(s, x(s)) d u(s)-\int_{0}^{t_{0}+} \frac{e^{s}-e^{2 t-s}}{2 e^{t}} g(s, x(s)) d u(s) \\
& +\int_{0}^{t_{0}+} \frac{e^{s}-e^{2 t-s}}{2 e^{t}} g(s, x(s)) d u(s)-\int_{0}^{t_{0}+} \frac{e^{s}-e^{2\left(t_{0}+\right)-s}}{2 e^{t_{0}+}} g(s, x(s)) d u(s) \mid \\
& \leq\left|\int_{0}^{1} \frac{\left(e^{t+\left(t_{0}+\right)}-1\right)\left(e^{t}-e^{t_{0}+}\right)\left(e^{2-s}+e^{s}\right)}{2\left(e^{2}-1\right) e^{t+\left(t_{0}+\right)}}(f(s, x(s))+x(s)) d s\right| \\
& +\left|\int_{0}^{1} \frac{\left(e^{t+\left(t_{0}+\right)}-1\right)\left(e^{t}-e^{t_{0}+}\right)\left(e^{2-s}+e^{s}\right)}{2\left(e^{2}-1\right) e^{t+\left(t_{0}+\right)}} g(s, x(s)) d u(s)\right| \\
& +\left|\int_{0}^{t_{0}+} \frac{\left(e^{t+\left(t_{0}+\right)-s}+e^{s}\right)\left(e^{t}-e^{t_{0}+}\right)}{2 e^{t+\left(t_{0}+\right)}}(f(s, x(s))+x(s)) d s\right| \\
& +\left|\int_{t}^{t_{0}+} \frac{e^{s}-e^{2 t-s}}{2 e^{t}}(f(s, x(s))+x(s)) d s\right| \\
& +\left|\int_{0}^{t_{0}+} \frac{\left(e^{t+\left(t_{0}+\right)-s}+e^{s}\right)\left(e^{t}-e^{t_{0}+}\right)}{2 e^{t+\left(t_{0}+\right)}} g(s, x(s)) d u(s)\right| \\
& +\left|\int_{t}^{t_{0}+} \frac{e^{s}-e^{2 t-s}}{2 e^{t}} g(s, x(s)) d u(s)\right| .
\end{aligned}
$$


By $\left(\mathrm{C}_{3}\right)$, we obtain

$$
\begin{aligned}
& \left|\int_{0}^{1} \frac{\left(e^{t+\left(t_{0}+\right)}-1\right)\left(e^{t}-e^{t_{0}+}\right)\left(e^{2-s}+e^{s}\right)}{2\left(e^{2}-1\right) e^{t+\left(t_{0}+\right)}}(f(s, x(s))+x(s)) d s\right| \\
& \leq\left|\frac{\left(e^{t}-e^{t_{0}+}\right)\left(e^{t+\left(t_{0}+\right)}-1\right)}{2\left(e^{2}-1\right) e^{t+\left(t_{0}+\right)}}\right|\left(\left|\int_{0}^{1}\left(e^{2-s}+e^{s}\right) q_{1}(s) d s\right|\right. \\
& \left.+\left|\int_{0}^{1}\left(e^{2-s}+e^{s}\right) q_{2}(s) d s\right|\right) \\
& \rightarrow 0, \text { as } t \rightarrow t_{0}+ \\
& \left|\int_{0}^{t_{0}+} \frac{\left(e^{t+\left(t_{0}+\right)-s}+e^{s}\right)\left(e^{t}-e^{t_{0}+}\right)}{2 e^{t+\left(t_{0}+\right)}}(f(s, x(s))+x(s)) d s\right| \\
& \leq\left|\frac{e^{t}-e^{t_{0}+}}{2 e^{t+\left(t_{0}+\right)}}\right|\left(\left|\int_{0}^{1}\left(e^{t+\left(t_{0}+\right)-s}+e^{s}\right) q_{1}(s) d s\right|\right. \\
& \left.+\left|\int_{0}^{1}\left(e^{t+\left(t_{0}+\right)-s}+e^{s}\right) q_{2}(s) d s\right|\right) \\
& \rightarrow 0, \text { as } t \rightarrow t_{0}+\text {; }
\end{aligned}
$$

and

$$
\begin{aligned}
& \left|\int_{t}^{t_{0}+} \frac{e^{s}-e^{2 t-s}}{e^{2 t}}(f(s, x(s))+x(s)) d s\right| \\
& \quad \leq\left|\int_{t}^{t_{0}+} \frac{e^{s}-e^{2 t-s}}{e^{2 t}} q_{1}(s) d s\right|+\left|\int_{t}^{t_{0}+} \frac{e^{s}-e^{2 t-s}}{e^{2 t}} q_{2}(s) d s\right| \\
& \quad \rightarrow 0, \text { as } t \rightarrow t_{0}+.
\end{aligned}
$$

By $\left(\mathrm{C}_{4}\right),\left(\mathrm{C}_{6}\right)$ and the Hölder inequality (Lemma 2.9), we get

$$
\begin{aligned}
& \left|\int_{0}^{1} \frac{\left(e^{t+\left(t_{0}+\right)}-1\right)\left(e^{t}-e^{t_{0}+}\right)\left(e^{2-s}+e^{s}\right)}{2\left(e^{2}-1\right) e^{t+\left(t_{0}+\right)}} g(s, x(s)) d u(s)\right| \\
& \quad \leq\left|\frac{\left(e^{t}-e^{t_{0}+}\right)\left(e^{t+\left(t_{0}+\right)}-1\right)}{\left(e^{2}-1\right) e^{t+\left(t_{0}+\right)}}\right|\left(e^{2}+1\right)\left(\left|g_{0}\right|+\bar{g}\right)\|u\| \\
& \quad \rightarrow 0, \quad \text { as } t \rightarrow t_{0}+
\end{aligned}
$$

and

$$
\begin{aligned}
& \left|\int_{0}^{t_{0}+} \quad \frac{\left(e^{t+\left(t_{0}+\right)-s}+e^{s}\right)\left(e^{t}-e^{t_{0}+}\right)}{2 e^{t+\left(t_{0}+\right)}} g(s, x(s)) d u(s)\right| \\
& \quad \leq\left|\frac{e^{t}-e^{t_{0}+}}{e^{t+\left(t_{0}+\right)}}\right|\left(e^{t+\left(t_{0}+\right)}+1\right)\left(\left|g_{0}\right|+\bar{g}\right)\|u\| \\
& \quad \rightarrow 0, \quad \text { as } t \rightarrow t_{0}+.
\end{aligned}
$$


Moreover,

$$
\begin{aligned}
& \left|\sum_{i=1}^{m} \frac{e^{\xi_{i}}-e^{2 t-\xi_{i}}}{2 e^{t}} g\left(\xi_{i}, x\left(\xi_{i}\right)\right)\left(u\left(t_{i}\right)-u\left(t_{i-1}\right)\right)\right| \\
& =\mid \frac{e^{t}-e^{2 t-t}}{2 e^{t}} g(t, x(t)) u(t)-\frac{e^{t_{0}+}-e^{2 t-\left(t_{0}+\right)}}{2 e^{t}} g\left(t_{0}+, x\left(t_{0}+\right)\right) u\left(t_{0}+\right) \\
& -\sum_{i=1}^{m}\left(\frac{e^{\xi_{i}}-e^{2 t-\xi_{i}}}{2 e^{t}} g\left(\xi_{i}, x\left(\xi_{i}\right)\right)-\frac{e^{\xi_{i-1}}-e^{2 t-\xi_{i-1}}}{2 e^{t}} g\left(\xi_{i-1}, x\left(\xi_{i-1}\right)\right)\right) u\left(t_{i-1}\right) \\
& =\mid\left(\frac{e^{t}-e^{2 t-t}}{2 e^{t}} g(t, x(t))-\frac{e^{t_{0}+}-e^{2 t-\left(t_{0}+\right)}}{2 e^{t}} g\left(t_{0}+, x\left(t_{0}+\right)\right)\right) u\left(t_{0}+\right) \\
& -\sum_{i=1}^{m}\left(\frac{e^{\xi_{i}}-e^{2 t-\xi_{i}}}{2 e^{t}} g\left(\xi_{i}, x\left(\xi_{i}\right)\right)-\frac{e^{\xi_{i-1}}-e^{2 t-\xi_{i-1}}}{2 e^{t}} g\left(\xi_{i-1}, x\left(\xi_{i-1}\right)\right)\right) u\left(t_{i-1}\right) \\
& =\mid \sum_{i=1}^{m}\left(\frac{e^{\xi_{i}}-e^{2 t-\xi_{i}}}{2 e^{t}} g\left(\xi_{i}, x\left(\xi_{i}\right)\right)\right. \\
& \left.-\frac{e^{\xi_{i-1}}-e^{2 t-\xi_{i-1}}}{2 e^{t}} g\left(\xi_{i-1}, x\left(\xi_{i-1}\right)\right)\right)\left(u\left(t_{i-1}\right)-u\left(t_{0}+\right)\right) \\
& \leq \operatorname{Var}_{\left[t_{0}+, t\right]} \frac{e^{s}-e^{2 t-s}}{2 e^{t}} g(s, x(s)) \sup _{s \in\left[t_{0}+, t\right]}\left|u(s)-u\left(t_{0}+\right)\right| \\
& \leq\left[\operatorname{Var}_{\left[t_{0}+, t\right]} \frac{e^{s}-e^{2 t-s}}{2 e^{t}}\left(\left|g_{0}\right|+\operatorname{Var}_{\left[t_{0}+, t\right]} g(s, x(s))\right)+\frac{e^{2 t-t_{0}+}-e^{t_{0}+}}{2 e^{t}} \operatorname{Var}_{\left[t_{0}+, t\right]} g(s, x(s))\right] \\
& \times \sup _{s \in\left[t_{0}+, t\right]}\left|u(s)-u\left(t_{0}+\right)\right| \\
& \leq \frac{e^{2}-1}{2 e}\left(\left|g_{0}\right|+2 \operatorname{Var}_{\left[t_{0}+, t\right]} g(s, x(s))\right) \sup _{s \in\left[t_{0}+, t\right]}\left|u(s)-u\left(t_{0}+\right)\right| \\
& \leq \frac{e^{2}-1}{2 e}\left(\left|g_{0}\right|+2 \bar{g}\right) \sup _{s \in\left[t_{0}+, t\right]}\left|u(s)-u\left(t_{0}+\right)\right| \\
& \rightarrow 0, \text { as } t \rightarrow t_{0}+\text {. }
\end{aligned}
$$

By Definition 2.7, we get

$$
\left|\int_{t}^{t_{0}+} \frac{e^{s}-e^{2 t-s}}{2 e^{t}} g(s, x(s)) d u(s)\right| \rightarrow 0, \text { as } t \rightarrow t_{0}+.
$$

Hence,

$$
\left|\mathcal{A} x(t)-\mathcal{A} x\left(t_{0}+\right)\right| \rightarrow 0, \text { as } t \rightarrow t_{0}+,
$$

independently of $x$. Similarly, for $t_{0} \in(0,1]$, we can prove that

$$
\left|\mathcal{A} x\left(t_{0}-\right)-\mathcal{A} x(t)\right| \rightarrow 0, \text { as } t \rightarrow t_{0}-\text {. }
$$

Therefore, $\mathcal{A}\left(B_{r}\right)$ is equiregulated on $[0,1]$ in terms of Definition 2.4

As a consequence of Steps 1 and 2 together with Lemma 2.5. we can conclude that the set $\mathcal{A}\left(B_{r}\right)$ is relatively compact in $G_{-}([0,1] ; \mathbb{R})$. 
Step 3. $\mathcal{A}$ is continuous on $B_{r}$.

Let $\left\{x_{n}\right\}_{n=1}^{\infty}$ be a convergent sequence on $B_{r}$ and $x_{n} \rightarrow x$ as $n \rightarrow \infty$.

By $\left(\mathrm{C}_{2}\right)$ and $\left(\mathrm{C}_{5}\right)$, we have

$$
\begin{aligned}
& f\left(\cdot, x_{n}(\cdot)\right) \rightarrow f(\cdot, x(\cdot)) \text { pointwise, as } n \rightarrow \infty, \\
& g\left(\cdot, x_{n}(\cdot)\right) \rightarrow g(\cdot, x(\cdot)) \text { uniformly, as } n \rightarrow \infty .
\end{aligned}
$$
have

According to $\left(\mathrm{C}_{3}\right)$ and the controlled convergence theorem of Lemma 2.11, we

$$
\lim _{n \rightarrow \infty} \int_{0}^{1} K(t, s) f\left(s, x_{n}(s)\right) d s=\int_{0}^{1} K(t, s) f(s, x(s)) d s,
$$

independently of $t \in[0,1]$. Furthermore, according to $\left(\mathrm{C}_{6}\right)$ and the convergence theorem of Proposition 2.10, we have

$$
\lim _{n \rightarrow \infty} \int_{0}^{1} K(t, s) g\left(s, x_{n}(s)\right) d u(s)=\int_{0}^{1} K(t, s) g(s, x(s)) d u(s), \quad t \in[0,1] .
$$

Therefore, $\lim _{n \rightarrow \infty} \mathcal{A} x_{n}(\cdot)=\mathcal{A} x(\cdot)$. This shows that $\mathcal{A}$ is continuous.

Thus, $\mathcal{A}$ satisfies the hypotheses of Lemma 3.3, and the application of Schauder's fixed point theorem shows that $\mathcal{A}$ has at least a fixed point which is a solution of the problem 1.1)-(1.2). This completes the proof.

Let us replace $\left(\mathrm{C}_{2}\right)$ and $\left(\mathrm{C}_{5}\right)$ by:

$\left(\mathrm{C}_{2}^{\prime}\right)$ There exists a constant $L_{1}>0$ such that

$$
|(f(\cdot, x(\cdot))+x(\cdot))-(f(\cdot, y(\cdot))+y(\cdot))| \leq L_{1}|x(\cdot)-y(\cdot)|, \quad \forall x, y \in G_{-}([0,1] ; \mathbb{R}) .
$$

$\left(\mathrm{C}_{5}^{\prime}\right)$ There exists a constant $L_{2}>0$ such that

$$
\underset{[0,1]}{\operatorname{Var}}[g(\cdot, x(\cdot))-g(\cdot, y(\cdot))] \leq L_{2}\|x-y\|, \quad \forall x, y \in G_{-}([0,1] ; \mathbb{R}) .
$$

Then we have the following uniqueness result.

The statement below is Banach's contraction principle.

Lemma 3.5. Let $(\mathbb{M}, d)$ be a nonempty complete metric space. If $\mathcal{T}: \mathbb{M} \rightarrow \mathbb{M}$ is a contraction mapping, i.e., $d(\mathcal{T} x, \mathcal{T} y) \leq q d(x, y)$ for some $0<q<1$ and all $x, y \in \mathbb{M}$, then exists a unique $\xi \in \mathbb{M}$ such that $\mathcal{T} \xi=\xi$.

With the help of this lemma we can now prove the uniqueness of solutions of problem (1.1)-(1.2).

Theorem 3.6. Under the assumptions $\left(C_{1}\right),\left(C_{2}^{\prime}\right),\left(C_{4}\right)$, and $\left(C_{5}^{\prime}\right)$, if

$$
M L_{1}+(4 M-2 m) L_{2}\|u\|<1
$$

then the problem (1.1)- 1.2 has a unique solution on $[0,1]$. 
Proof. We shall show that $\mathcal{A}$ defined by 3.15 is a contraction. Indeed, let $x_{1}, x_{2} \in$ $G_{-}([0,1] ; \mathbb{R})$. Then, for each $t \in[0,1]$, we have

$$
\begin{aligned}
& \left|\mathcal{A} x_{1}(t)-\mathcal{A} x_{2}(t)\right| \\
& \leq\left|\int_{0}^{1} K(t, s)\left[\left(f\left(s, x_{1}(s)\right)+x_{1}(s)\right)-\left(f\left(s, x_{2}(s)\right)+x_{2}(s)\right)\right] d s\right| \\
& +\left|\int_{0}^{1} K(t, s)\left(g\left(s, x_{1}(s)\right)-g\left(s, x_{2}(s)\right)\right) d u(s)\right| \\
& \leq\left|\int_{0}^{1} K(t, s) L_{1}\right| x_{1}(s)-x_{2}(s)|d s|+\left[\left|K(t, 0)\left(g\left(0, x_{1}(0)\right)-g\left(0, x_{2}(0)\right)\right)\right|\right. \\
& +\left|K(t, 1)\left(g\left(1, x_{1}(1)\right)-g\left(1, x_{2}(1)\right)\right)\right| \\
& \left.+\operatorname{Var}_{[0,1]} K(t, s)\left(g\left(s, x_{1}(s)\right)-g\left(s, x_{2}(s)\right)\right)\right]\|u\| \\
& \leq \int_{0}^{1}|K(t, s)| L_{1}\left|x_{1}(s)-x_{2}(s)\right| d s+\left[|K(t, 1)| \underset{[0,1]}{\operatorname{Var}}\left(g\left(s, x_{1}(s)\right)-g\left(s, x_{2}(s)\right)\right)\right. \\
& +\underset{[0,1]}{\operatorname{Var}} K(t, s) \underset{[0,1]}{\operatorname{Var}}\left(g\left(s, x_{1}(s)\right)-g\left(s, x_{2}(s)\right)\right) \\
& \left.+M \underset{[0,1]}{\operatorname{Var}}\left(g\left(s, x_{1}(s)\right)-g\left(s, x_{2}(s)\right)\right)\right]\|u\| \\
& \leq L_{1} M\left\|x_{1}-x_{2}\right\|+\left[\left(2 M+\underset{[0,1]}{\operatorname{Var} K(t, s))} \underset{[0,1]}{\operatorname{Var}}\left(g\left(s, x_{1}(s)\right)-g\left(s, x_{2}(s)\right)\right)\right]\|u\|\right. \\
& \leq L_{1} M\left\|x_{1}-x_{2}\right\|+(4 M-2 m) L_{2}\left\|x_{1}-x_{2}\right\|\|u\| \\
& \leq\left[M L_{1}+(4 M-2 m) L_{2}\|u\|\right]\left\|x_{1}-x_{2}\right\| .
\end{aligned}
$$

Let

$$
L:=M L_{1}+(4 M-2 m) L_{2}\|u\| .
$$

Thus, by (3.18), for $0<L<1$, we obtain

$$
\left\|\mathcal{A} x_{1}-\mathcal{A} x_{2}\right\| \leq L\left\|x_{1}-x_{2}\right\| .
$$

This implies that $\mathcal{A}$ is a contraction. We deduce by Banach's contraction principle that $\mathcal{A}$ has a unique fixed point which is a unique solution of the problem (1.1)(1.2). This completes the proof.

\section{EXAMPLES}

In this section, we give two examples to illustrate Theorem 3.4 and Theorem 3.6

Example 4.1. Consider the boundary value problem:

$$
\left\{\begin{array}{l}
-D^{2} x=\sin (x)-x+H\left(t-\frac{1}{2}\right) D u, \quad t \in[0,1] \\
D x(0)=D x(1)=0
\end{array}\right.
$$


where

$$
u(t)= \begin{cases}W(t), & 0 \leq t<\frac{1}{4} \\ H\left(t-\frac{1}{2}\right), & \frac{1}{4} \leq t \leq 1\end{cases}
$$

$W(t)$ is the Weierstrass function $W(t)=\sum_{n=1}^{\infty} \frac{\sin 7^{n} \pi t}{2^{n}}$ (see [4]), and $H(t)$ is the Heaviside function, i.e.,

$$
H(t)= \begin{cases}1, & t \geq 0 \\ 0, & t<0 .\end{cases}
$$

It is well known that $W(t)$ is continuous but pointwise differentiable nowhere on $[0,1]$ and $H(t)$ is of bounded variation but not continuous on $[0,1]$, hence $u(t)$ is only a regulated function.

Let

$$
f(t, x)=\sin (x)-x, \quad g(t, x)=H\left(t-\frac{1}{2}\right), \quad t \in[0,1] .
$$

Since $g_{0}=0, \bar{g}=1$ and $q_{1} \equiv-1, q_{2} \equiv 1, t \in[0,1]$, we have that assumptions $\left(\mathrm{C}_{1}\right)-$ $\left(\mathrm{C}_{6}\right)$ are satisfied. Moreover, $\|H\|=1,\|W\| \leq \sum_{n=1}^{\infty} \frac{1}{2^{n}}=1$ and thus $\|u\| \leq 1$. Hence,

$$
r=M Q+(4 M-2 m)\left(\left|g_{0}\right|+\bar{g}\right)\|u\| \leq 6 M-2 m=\frac{2\left(3 e^{2}+3-2 e\right)}{e^{2}-1} .
$$

Therefore, the existence of a solution of problem (4.1) is guaranteed by Theorem 3.4

Example 4.2. Consider the boundary value problem:

$$
\left\{\begin{array}{l}
-D^{2} x=\frac{1}{3} \sin (x)-x+2 t \sin \left(t^{-2}\right)-\frac{2}{t} \cos \left(t^{-2}\right)+H\left(t-\frac{2}{3}\right) D H\left(t-\frac{2}{3}\right), \\
D x(0)=D x(1)=0, \quad t \in[0,1],
\end{array}\right.
$$

where $H(t)$ is the Heaviside function 4.2 .

Let

$$
\begin{aligned}
& f(t, x)=\frac{1}{3} \sin (x)-x+2 t \sin \left(t^{-2}\right)-\frac{2}{t} \cos \left(t^{-2}\right), \\
& g(t, x)=u(t)=H\left(t-\frac{2}{3}\right), \quad t \in[0,1] .
\end{aligned}
$$

If $L_{1}=\frac{1}{3}$, then $f$ satisfies $\left(\mathrm{C}_{1}\right),\left(\mathrm{C}_{2}^{\prime}\right)$.

On the other hand, since $g_{0}=0, L_{2}=\frac{1}{4 e^{2}+4-4 e}$, and $\bar{g}=1$, we have that $g$ satisfies $\left(\mathrm{C}_{4}\right)$ and $\left(\mathrm{C}_{5}^{\prime}\right)$. Moreover, $\|u\|=\|H\|=1$. Hence,

$$
L=M L_{1}+(4 M-2 m) L_{2}\|u\|=\frac{e^{2}+4}{3\left(e^{2}-1\right)}<1 .
$$

Therefore, the uniqueness of solution of problem 4.3 is guaranteed by Theorem 3.6

Remark 4.3. In Example 4.1, $u(t)$ is neither continuous nor of bounded variation but it is regulated, so $H\left(t-\frac{1}{2}\right)$ is not Lebesgue-Stieltjes or Riemann-Stieltjes integrable with respect to the regulated function $u(t)$. In Example $4.2, f(t, x)$ 
is a highly oscillating function, which is not Lebesgue integrable. The function $g(t, x)=u(t)=H\left(t-\frac{2}{3}\right)$ is of bounded variation but not continuous; $g(t, x)$ and $u(t)$ have a common point of discontinuity at $t=\frac{2}{3}$, so $g(t, x)$ is not Riemann-Stieltjes integrable with respect to $u(t)$. However, in the two examples, the HenstockKurzweil-Stieltjes integral is valid. This implies that our results are more extensive.

\section{REFERENCES}

[1] A. Cañada, J.A. Montero, S. Villegas, Liapunov-type inequalities and Neumann boundary value problems at resonance. Math. Inequal. Appl. 8 (2005), no. 3, 459-475. MR 2148238

[2] P.C. Das, R.R. Sharma, Existence and stability of measure differential equations. Czechoslovak Math. J. 22(97) (1972), 145-158. MR 0304815

[3] D. Fraňková, Regulated functions. Math. Bohem. 116 (1991), no. 1, 20-59. MR 1100424

[4] G.H. Hardy, Weierstrass's non-differentiable function. Trans. Amer. Math. Soc. 17 (1916), no. 3, 301-325. MR 1501044.

[5] P. Krejčí, P. Laurençot, Generalized variational inequalities. J. Convex Anal. 9 (2002), no. 1, 159-183. MR 1917394

[6] P.Y. Lee, Lanzhou Lectures on Henstock Integration. World Scientific, Singapore, 1989. MR 1050957

[7] G.A. Monteiro, A. Slavík, M. Tvrdý, Kurzweil-Stieltjes Integral. World Scientific, Singapore, 2019. MR 3839599

[8] G.A. Monteiro, B. Satco, Distributional, differential and integral problems: equivalence and existence results. Electron. J. Qual. Theory Differ. Equ. 2017, Paper No. 7, 26 pp. MR 3606985

[9] S. Sánchez-Perales, J. F. Tenorio, Laplace transform using the Henstock-Kurzweil integral. Rev. Un. Mat. Argentina 55 (2014), no. 1, 71-81. MR 3264685

[10] S. Sánchez-Perales, The initial value problem for the Schrödinger equation involving the Henstock-Kurzweil integral. Rev. Un. Mat. Argentina 58 (2017), no. 2, 297-306. MR 3733209

[11] B. Satco, Regulated solutions for nonlinear measure driven equations. Nonlinear Anal. Hybrid Syst. 13 (2014), 22-31. MR 3209695

[12] Š. Schwabik, G. J. Ye, Topics in Banach space Integration. World Scientific, Singapore, 2005. MR 2167754

[13] J.P. Sun, W.T. Li, Multiple positive solutions to second-order Neumann boundary value problems. Appl. Math. Comput. 146 (2003), no. 1, 187-194. MR 2007778

[14] E. Talvila, The regulated primitive integral. Illinois J. Math. 53 (2009), no. 4, 1187-1219. MR 2741185

Guoju Ye, Mingxia Zhang ${ }^{凶}$, Wei Liu

College of Science, Hohai University, Nanjing 210098, China

yegj@hhu.edu.cn

mingxiazhangzmx@163.com

liuw626@hhu.edu.cn

Dafang Zhao

College of Science, Hohai University, Nanjing 210098, China; School of Mathematics and

Statistics, Hubei Normal University, Hubei 435002, China

dafangzhao@163.com

Received: April 24, 2018

Accepted: March 2, 2019

Rev. Un. Mat. Argentina, Vol. 60, No. 2 (2019) 